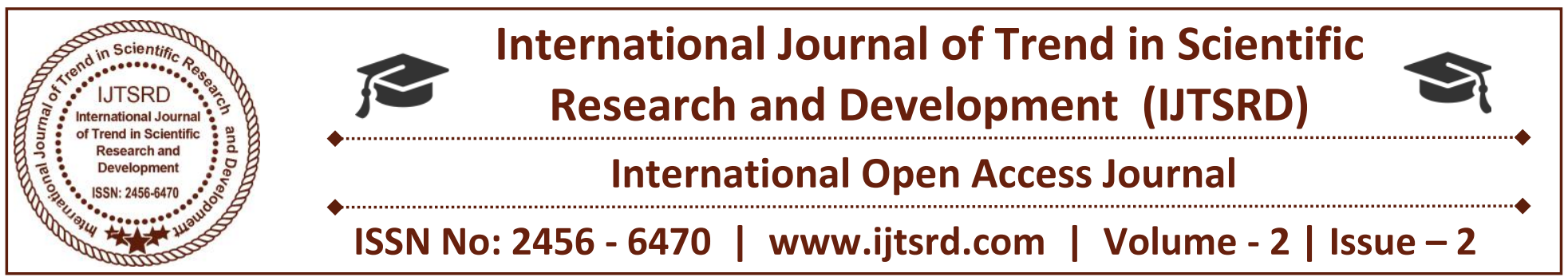

\title{
Advanced Crop Monitoring using Internet of Things based Smart Intrusion \& Prevention in Agricultural Land
}

\author{
G.Naveen Balaji ${ }^{1}$, V.Nandhini ${ }^{2}$, S.Mithra ${ }^{2}$, R.Naveena ${ }^{2}$, N.Priya ${ }^{2}$ \\ ${ }^{1}$ Assistant Professor, ECE Department, SNS College of Technology, Coimbatore, India \\ ${ }^{2}$ UG Student, ECE Department, SNS College of Technology, Coimbatore, India
}

\section{ABSTRACT}

In the world, economic growth of many countries mainly depends on crop cultivation. Agriculture provides the food for people and produces several raw materials for industries. Now-a-days, crop cultivation is decreasing rapidly. To overcome this problem, monitoring the crop water requirements, temperature and humidity of the cultivating land and a system to prevent the entry of animals into the farm is required. The main concept of this paper is to prevent the animal intrusion and to monitor the crop water requirements. This method increases the crop yield which will not be harmful to the cultivation as well as to the animals. This system mainly aims to secure the crops by the deployment of sensor and prevent intrusion of animals using image processing technique.

Keywords: Arduino, IoT, Sensors, Video surveillance

\section{INTRODUCTION}

Agriculture is the backbone of our country. To improve productivity in agricultural lands modern crop yielding methods have been used. Smart sensing and image processing methods are used to increase the crop productivity and to prevent damages made by intrusion of animals. It is necessary to measure the crop water requirements, temperature and humidity in agricultural lands which can be done using wireless sensor networks. The internet of things (IoT) along with the sensor network helps the farmers to stay connected to his field from anywhere and anytime. Prevention of animal intrusion is the most important thing to secure the crops. In this paper, we propose a method of image processing technique to prevent the animal intrusion which is done using MATLAB processing. The image captured is classified and segmented and compared with the previously stored images. The farmer will be able to view the information of his field through webpage and will be alerted through message when any intrusion occurs. Similarly to monitor the soil water requirements sensors are deployed in the farmland.

\section{IE LITERATURE REVIEW}

A system by using the wireless sensor networks to detect the intrusion of birds and animals in the agricultural lands is discussed [3]. The animal faces are detected by area unit measurement by utilizing face notification technique with totally different native distinction configuration of effulgence channel to detect the image region of animal faces. The infrared sensor is used detect the entering of the animals. This paper has the lighting drawback, in which a sudden amendment of lighting impact largely in indoor application will have an effect in the presence of animal intrusion. The brightness level drawback with changes of natural surroundings from day to night time at outside closed-circuit television may effect on the detection. The moving leaves by wind may be considered foreground image and a few inactive animals that stay static time as background image by the algorithms. 
For crop monitoring, a method of intelligent agricultural crop monitoring system based on ZigBee technology is discussed here [5]. The system involves the processing of data acquisition, processing, and transmission and reception functions. The main purpose is to maintain the system efficiency in the monitoring area. IOT technology is used in this monitoring system with the B-S structure and cc2530 as like a chip processing for wireless sensor node. But this paper does not have the technique of predicting crop water requirement.

This system explains the use of pyro electric infrared sensors. It produces an electric potential by means of a very small change in temperature [8]. PIR sensors are quite fit for detecting moving targets. Many security systems have made PIR sensors to be a good alarm for intrusion and precise counter for targets which are not only for people but also for vehicles and so on. PIR sensors are not deployed in an indoor environment where the detecting range is confined in several meters. They are used to detect target more than 20 meters away in unattended wild ground environment. This explains a system to monitor a specific wild district using PIR sensors. In this paper recognition of more kinds of targets is not implemented and the accuracy of classifying is sometimes disturbed by the use of sensors. This drawback can be overcome by using certain algorithms instead of time domain and frequency domain.

This system explains the use of Python scripts for integrating the Internet of things, Raspberry pi, and wireless sensor networks to accentuate the methods for identification of rodents, threats to crops and delivering real time notification based on information analysis and processing without human intervention [9]. The lack of information transmission and data analyzing has been solved by integration of internet of things with currently available security devices in order to achieve efficient food preservation and productivity. This paper does not have the technique of pattern recognition for machine learning and to identify objects and categorize them into humans, rodents and animals, also sensor fusion can be done to increase the functionality of the device. This drawback can be overcome by the grid of panels consisting PIR sensors and URD sensors.

\section{PROPOSED WORK}

The image processing using video surveillance and embedded system using the sensors are taken into consideration for monitoring area. The crop water requirement and temperature, humidity values are measured by using the sensors. The average value of temperature and humidity of the surrounding and the soil moisture measured is sent to the arduino UNO R3. This average value from the controller is displayed in digital form in the webpage.

The animal intrusion into the agricultural land is detected by image processing technique. It is based on the movement detection of the animal using the continuous video surveillance through the cameras placed. The cameras are fixed in multiple areas in the agricultural land. The images of the animals are already stored in the database. If any animal is intruded into the land, the image of the entered animal is captured. The captured image is then resized to the defined size and then it is converted into the gray scale image to detect the foreground image of interest. Now the converted image is compared with the previously stored images. If both the images are matched then an alert message will be sent to the person through GSM module.

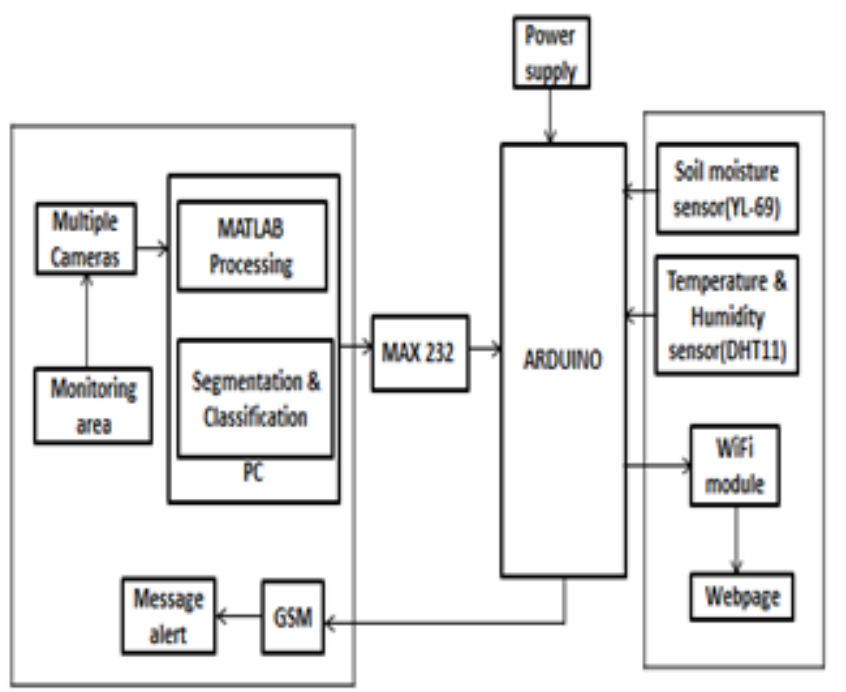

Fig 1: Block diagram of proposed system

\section{HARDWARE USED}

\section{A. ARDUINO MICROCONTROLLER}

\section{ATMEGA 328}

A single chip microcontroller ATmega328 is created by the family of megaAVR. A $32 \mathrm{kB}$ ISP flash memory with read write capabilities are combined by 
the 8-bit AVR RISC based microcontroller. The compare modes are used in general purpose I/O lines, 32 general purpose working registers and flexible timer/counter modes are used. The arduino UNO R3 has 14 digital I/O pins and 6 analog pins. A frequency created by crystal oscillator is $16 \mathrm{MHz}$. To start the arduino AC-DC adapter otherwise a battery is used. It differs from all the existing boards so the FTDI USBserial drive chip is not used. The 1.0 version of arduino is used as a reference. The operating voltage range of UNO R3 is $1.8-5.5$ volts.

\section{B. SOIL MOISTURE SENSOR YL-69}

It is also called as hygrometer. The level of humidity in the soil is measured using this sensor. The electronic board and probe which consists of two pads are the two different parts of the hygrometer. These are used to measure the moisture level of the soil in an agricultural land. A potentiometer is built in within the sensor and the output will be in digital form. The output value changes accordingly to the moisture level of the soil content. Depending on the moisture the output can be varied in digital as HIGH or LOW. The voltage decreases if the soil is in wet condition and low it is in dry condition.

\section{TEMPERATURE AND HUMIDITY SENSOR DHT-11}

It is made of three parts: humidity sensor which is resistive type, 8-bit microcontroller and a thermistor. These three are used to convert the analog signal into the digital form. The voltage varies between $3.3 \mathrm{v}$ and $5.5 \mathrm{v}$. The three pins used in the humidity sensors are VCC, ground, and data out.

\section{GSM MODULE}

SIM800 quad band GSM module is used. The range of frequency for SIM800 is GSM $850 \mathrm{MHz}$, EGSM $900 \mathrm{MHz}$, DCS $1800 \mathrm{MHz}$ and PCS $1900 \mathrm{MHz}$. It is $24 * 24 * 3 \mathrm{~mm}$ small configuration. It is used in all the requirements such as M2M, smart phone, PDA and other mobile devices. The current consumption is 1.2 MA as lower than other modules.

\section{E. Wi-Fi MODULE}

The type of module used here is ESP8266 and consist of TCP/IP protocol stack. A 32 bit microcontroller type is used in ESP8266. The CPU varies its frequency range from $80 \mathrm{MHz}$ to $160 \mathrm{MHz}$. The over clocking can double the CPU and flashback speed.

\section{F. MAX232}

A conversion of a signal TIA-232 serial port for compatible TTL logic circuits is made by the integration of a MAX232. This can act as a transmitter/dual receiver for the conversion of RX, TX, CTS, RTS signals. The inputs may be as high as 25 volts and the receiver decreases the TIA-232 inputs.

\section{SOFTWARE USED}

\section{G. ARDUINO}

Arduino can be used for the manufacturing of single board microcontroller and microcontroller kits. A digital devices and interactive objects built by arduino are used for sensing the control objects in the physical world. The CPU models in the arduino are Atmel AVR (8-bit), ARM cortex -M0+(32-bit), ARM cortex-M3(32-bit) and Intel Quark(X86)(32-bit). The built in memory for the arduino is SRAM. A arduino board is pre-assembled and the Arduino programming simplified from the $\mathrm{c} / \mathrm{c}++$ languages is called as "sketches" by the arduino.

\section{H. MATLAB}

A (2D) image is a two dimensional array of pixels. Most of the common image operations are manipulations of this two dimensional data. Matlab offers a variety of library functions for vector and matrix (2d array) manipulations. Thus it is used for quick prototyping and testing methods for image processing algorithms. It is possible to write algorithm in Matlab, as in $\mathrm{C}++$ or Java programming. Motion detection is a computer technology related to computer vision and image processing that deals with detecting instances of objects such as humans, animals, buildings, or cars in digital images and videos. Well-researched domains of object detection include face detection and pedestrian detection. Object detection has applications in many areas of computer vision, including image retrieval and video surveillance. Every class has its own special feature that helps in classifying the class. Object class detection uses these special features. A similar technique is used for face identification where eyes, nose, and lips can be found and features like skin color and distance between eyes can be found. 
International Journal of Trend in Scientific Research and Development (IJTSRD) ISSN: 2456-6470

\section{SYSTEM IMPLEMENTATION}

\section{PROGRAMMING IN ARDUINO}

Select ArduinoUNO from Tools>Board menu. A boot loader in arduino allows uploading the new code without the use of external hardware components. The mega8U2 is activated by connecting the solder jumper on the back of the board loaded with a DFU boot loader and resetting the 8U2.Atmel's FLIP software program is also used to load a new firmware.

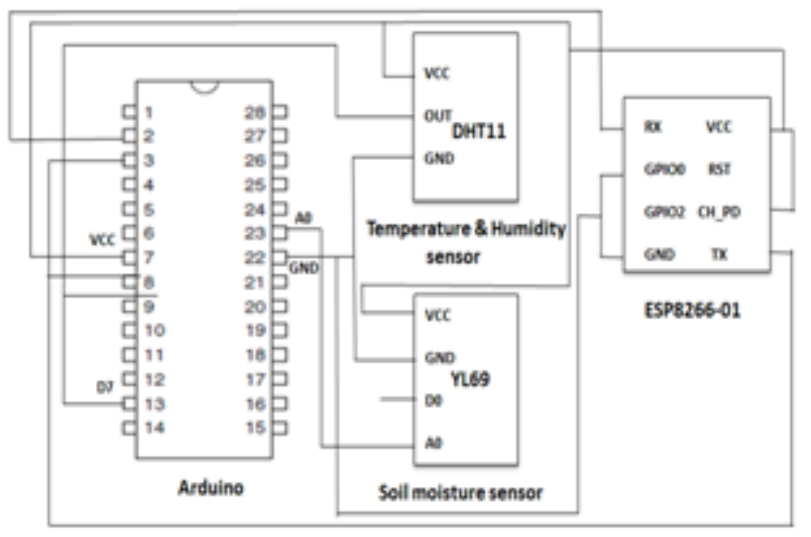

Fig 2: Circuit connections of sensors and Wi-Fi module with arduino

\section{SOIL MOISTURE SENSOR (YL-69)}

A0, D0, VCC and GND are the three pins available insoil moisture sensor.

Step 1: A0 is the analog input pin connected to the A0 of arduino.

Step 2: $5 \mathrm{~V}$ power supply of arduino is connected to the VCC of YL-69.

Step 3: Ground pin of the arduino is given to the ground pin of the sensor.

Step 4: The compilation of code is made to find out the errors.

Step 5: The code is uploaded to the arduino board.

\section{TEMPERATURE AND HUMIDITY SENSOR (DHT-11)}

Positive, output and negativepins are present in temperature and humidity sensor.

Step 1: 5V power supply of arduino is connected with positive pin of DHT 11.

Step 2: Digital output pin D7 of arduino is connected with output pin of DHT 11.

Step 3: The ground pin of arduino is connected with negative pin of DHT-11.
Step 4: The compilation of code is made to find out the errors.

Step 5: The code is uploaded to the arduino board.

\section{Wi-Fi MODULE (ESP8266-01)}

The ESP8266-01 has eight pins namely: RX, TX,CHPD, GPIO0, GPIO2, GND, and RESET.

Step 1: The RX pin of Wi-Fimodule (ESP8266-01) is connected to the RX pin of arduino UNO.

Step 2: The TX pin of Wi-Fimodule (ESP8266-01) is connected to the TX pin of arduino UNO.

Step 3: The VCC and CH_PD pins of Wi-Fimodule (ESP8266-01) is connected to VCC of arduino.

Step 4: The GPIO0 and GND pins of Wi-Fimodule (ESP8266-01) is connected to the GND pin of arduino.

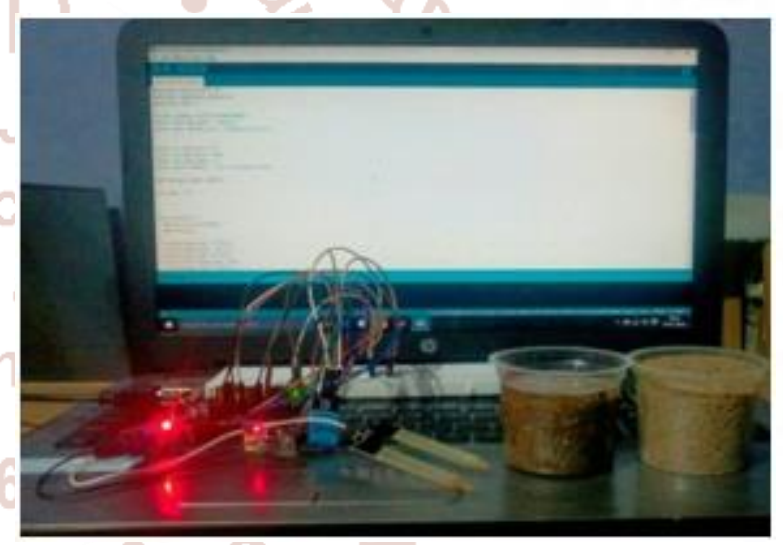

Fig 3: Hardware setup

\section{EXPERIMENTAL RESULT}

\section{SENSOR OUTPUT}

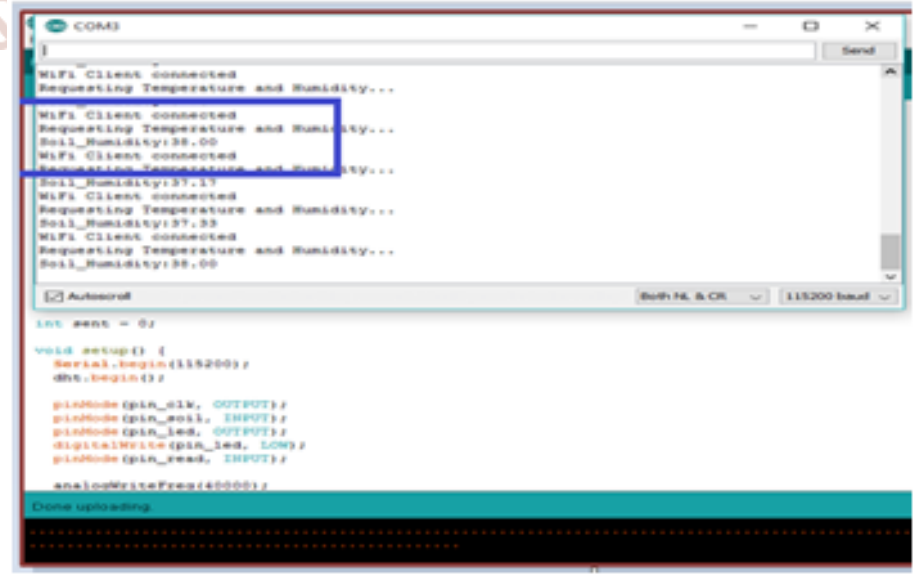

Fig 4: Average value of soil moisture and temperature and humidity displayed in the webpage 
Above is the serial monitor output of Arduino when the Wi-Fi client gets connected.

When the Wi-Fi gets connected it request the average value of temperature and humidity and the soil moisture and it is displayed in the webpage.

\section{IMAGE PROCESSING OUTPUT}

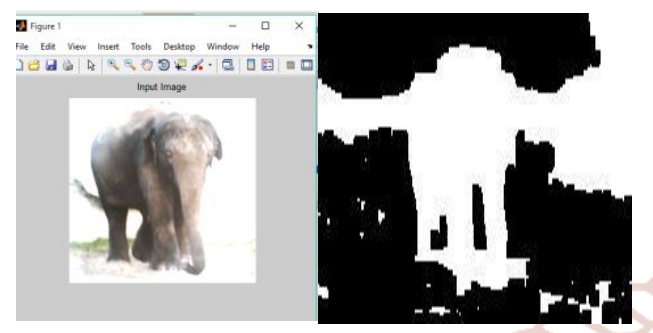

Fig 5: Input image Fig 6: Grayscale image

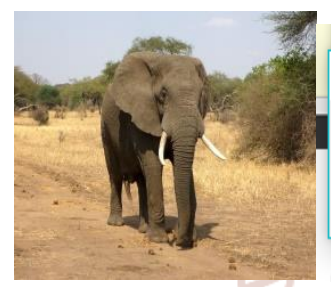

Fig 7: Database image

Fig 8: Final output
VIII. CONCLUSION AND FUTURE WORK

In this paper, we proposed a method for crop monitoring to increase the efficiency of the crop productivity by using the IOT technology and to avoid the damaging of crops by preventing from animal intrusion which can be monitored by using the multiple cameras in agricultural land. This paper is limited only for crop monitoring and thus it can be extended for automated water supply to the farm and also intrusion detection is limited only for two animals, hence in future this system can be enhanced with more animal detection with some advanced algorithms.

\section{REFERENCES}

1. Azhar, Hoque. S., Deravi.F.: "Automatic identification of wildlife using local binary patterns". IET Conf. on Image Processing (IPR), London, July 2012, pp. 1-6.

2. Balaji Bhanu, Raghava Rao, J.V.N. Ramesh and Mohammed Ali hussain, "Agriculture Field Monitoring and Analysis using Wireless Sensor Networks for improving Crop Production”, 2014
Eleventh International Conference on Wireless and Optical Communications Networks.

3. .Dr.P.UmaMaheswariPh.D, Anjali Rose Rajan, "Animals intrusion detection system using wireless sensor networks", 2016 Tenth international journal of advanced research in biology engineering science and technology.

4. Jing Dong, Johny Gary, Burnham Boots, Glen Rains, Frank Dellaert, "4D Crop Monitoring :Spatio - Temporal Reconstruction for Agriculture", 2017 IEEE International Conference on Robotics and Automation(ICRA), May 29-June 3, 2017.

5. LIU Dan, Cao Xin, Huang chongwei, JI Liang , “ Intelligent agent greenhouse environment monitoring system based on Internet of Things", 2015 International Conference on Intelligent Transportayion, Big Data and Smart city.

6. NagarajuAndavarappu and ValliKumariVatasvay, "Wild-Animal Recognition in Agriculture Farms Using W-COHO for Agro-Security", November 9,2017 ${ }^{\text {th }}$ International Journal of Computational Intelligence Research ISSN 0973-1873.

7. C P.Rajalakshmi and S.D. Mahalakshmi, "IOT Based Crop-Field Monitoring and Irrigation Automation",10th Int'1 Conf. Intelligent Systems and Control (ISCO), 2016, pp. 1-5.

8. Pampapathi B S, Manjunath $\mathrm{P}$ C, "Intrusion Detection Using Passive Infrared Sensor (PIR)", $7^{\text {th }}$ May 2016, Asian Journal of Engineering and Technology Innovation.

9. R.Radha, K.Kathiravan, V.Vineeth, J.Sanjay, S.Venkatesh, "Prevention Of Monkey Trespassing in Agricultural Field Using Agricultural Specific Flooding Approach in Wireless Sensor Networks", 2015 IEEE International Conference on Technological Innovations in ICT Agriculture and Rural Development.

10. Tanmay Baranwal, Nitika, Pushpendra kumar pateriya, "Development of IOT based Smart Security and Monitoring Devices for Agriculture", $2016^{\text {th }}$ International Conference -Cloud System and Big Data Engineering(Confluence). 